\title{
Evaluación del impacto del programa de vigilancia y promoción del desarrollo integral del niño sobre la estimulación en el hogar en niños menores de 3 años
}

Silvia Clementina Izazola Ezquerro, ${ }^{1}$ Rolando Rivera González, ${ }^{1}$ María Yadira Villanueva Romero, ${ }^{2}$ Luz Angélica Orozco Restrepo, ${ }^{2}$ José Antonio Sierra Cedillo y ${ }^{1}$ Rosa María Nájera ${ }^{2}$

\section{Introducción}

La promoción del desarrollo infantil plantea formas integrales de cuidado y crianza que contemplan la orientación sobre higiene, nutrición, reducción de exposición a contaminantes, estrategias de prevención de accidentes y estilos de crianza psicosociales adecuados. Se destacan las formas de interacción del niño con sus padres, cuidadores y el ambiente familiar, que pueden ser abordadas por los promotores de salud (Sánchez, Rivera, y Figueroa, 2009). Esta perspectiva de promoción se integra a la estrategia denominada Sistema de Vigilancia y Promoción del Desarrollo Integral del Niño (SIVIPRODIN), propuesta del grupo de trabajo del Laboratorio de Seguimiento del Neurodesarrollo del INP (Bronfenbrenner, 1999). Señala que desde fases tempranas, el desarrollo humano toma lugar a través de procesos de interacción recíproca progresivamente más complejas entre un ser

1 Instituto Nacional de Pediatría.

2 Universidad Autónoma Metropolitana-X. 
humano biopsicosocial activo y las personas, objetos y símbolos en su medio ambiente externo inmediato. La interacción debe darse de forma sostenida a lo largo de un periodo de tiempo. Tales formas duraderas de interacción en el medio ambiente inmediato son referidas como procesos proximales. Ejemplos de estos patrones duraderos son las actividades padres-niño, niño-niño, o a través del juego. Las características del proceso proximal que afectan el desarrollo varían sistemáticamente como función tanto de las características de la persona en desarrollo, como de su medio ambiente inmediato, donde los procesos toman lugar (Bronfenbrenner, 1999). Los procesos proximales han sido evaluados a través de los niños y sus interacciones típicas con objetos, materiales y personas dentro de su microsistema más común, que es el hogar. Una manera de medirlos es el Inventario de Estimulación en el Hogar HOME (Tudge, Oderob, Hoganc y Etz, 2003 y Tudge, 2009).

El presente trabajo buscar evaluar el efecto de la estrategia del SIVIPRODIN sobre las acciones que realizan los cuidadores en casa para estimular a los niños, identificando en qué medida se están logrando los objetivos para los que fue creado el programa y detectar áreas de oportunidad que permitan la toma de decisiones para mejorar su efectividad (Ángeles, 2011).

\section{Problema}

Los programas de vigilancia y promoción del desarrollo aplican estrategias para favorecer el desarrollo del niño a través de la incorporación de conceptos y prácticas referentes a las actividades de estimulación en el hogar que debe recibir el niño por parte de su madre o cuidador principal. Se espera que una vez incorporados estos comportamientos a nivel familiar se observen diferencias en el grado de estimulación con respecto a los niños y familias que no han sido intervenidos por el programa. Estas intervenciones buscan finalmente evidenciar y comprobar que existe una asociación importante entre las acciones que modifican el ambiente de manera positiva y el desarrollo de las 
diferentes potencialidades del niño, además de influir sobre los estilos de crianza y formas de cuidado.

\section{Justificación}

Una problemática que se presenta en los países en desarrollo, incluyendo México, como un factor asociado a la pobreza, es el limitado involucramiento de los padres en actividades cognitivamente estimulantes con sus hijos. El SIVIPRODIN es una estrategia de vigilancia y promoción del desarrollo aplicable al primer nivel de atención, posibilitando contactos más tempranos con la población infantil y sus familias, y promoviendo el desarrollo y estilos de crianza saludables. Como otros programas de este tipo, con miras a su aplicación más amplia, se requiere conocer el efecto que tiene sobre la organización del hogar y los cuidadores más cercanos para favorecer el desarrollo de las potencialidades en el niño a través de instrumentos como el HOME; para evaluar el cumplimiento de objetivos del programa; y para observar si se favorecen formas de estimulación en el hogar y organización del ambiente como promotoras del desarrollo infantil; por lo que se esperaría una variación significativa en los resultados obtenidos después de la exposición al programa.

\section{Objetivo}

Determinar el impacto de las estrategias de vigilancia y promoción del desarrollo del niño menor de 3 años de edad sobre la estimulación en el hogar.

\section{Método}

Es un estudio observacional, comparativo, transversal, prospectivo.

Población: Niños y niñas menores de 3 años de edad, de bajo riesgo, y sus madres o cuidador principal, residentes en una 
colonia de la Delegación Iztapalapa, expuestos al programa de promoción del desarrollo y crianza saludables (SIVIPRODIN). A través de un muestreo simple por cuotas, se incluyeron 162 niños (niños y sus cuidadores) menores de 36 meses; 78 de ellos expuestos al programa SIVIPRODIN y 84 casos no expuestos formaron parte del grupo control. Para evaluar el impacto del programa en los niños y sus familias se utilizó el instrumento Inventario HOME-IT. Los resultados de la estimulación en el hogar y sus seis subescalas (respuesta emocional y verbal de la madre, aceptación de las conductas del niño, organización del ambiente físico y temporal, disposición de los materiales apropiados de juego, capacidad de la madre para involucrarse con el niño y variedad de oportunidades para la estimulación diaria) fueron organizados de manera cuantitativa como la proporción de aciertos para la escala total y cada una de las subescalas que contiene el formato, y de forma cualitativa ordinal, formándose tres grupos en cada subescala, y la escala total (alta, media y baja estimulación). Se compararán las diferencias de medias en las puntuaciones total y por subescala del inventario HOME, después la frecuencia de baja media y alta estimulación en el grupo de expuestos y no expuestos. Se determinó si el programa tenía un impacto adecuado y satisfactorio en los efectos que valora el HOME, evaluando simultáneamente otras variables para determinar si el impacto es diferencial a determinadas condiciones sociodemográficas y económicas, tanto al grupo expuesto -al menos cuatro meses acudiendo al programa-, como al grupo control.

\section{Resultados}

Al contrastar al grupo de expuestos con el grupo control, se encontró que ambos grupos pertenecientes a la misma comunidad tienen características homólogas en cuanto a la edad de los niños, años concluidos de estudio de los cuidadores, el orden de nacimiento del niño y el estado civil, en las que no se observan diferencias estadísticamente significativas en los atributos que se presentan. Se encontraron diferencias estadísticamente 
significativas por sexo $\left(0.0494^{*}\right)$ (más hombres en los expuestos) y por edad de los cuidadores (0.0130*) (más jóvenes en los expuestos), considerándose que tales características eran compensadas por otras.

Siendo los grupos básicamente comparables en cuanto a sus principales características sociodemográficas, se aplicó la estrategia central de investigación consistente en saber si existen diferencios en las puntuaciones totales y por subescalas en el Inventario Home según los niños y sus familias estuviesen o no expuestos al programa. Se encontraron diferencias estadísticamente significativas en los puntajes totales del instrumento HOME (puntajes promedio en niños expuestos de $30.17 \pm 0.66$ $v s$ no expuestos promedio de $24.48 \pm 0.63$ ), en la subescala de organización promedio mayores en expuestos $(4.47 \pm 0.12)$ vs no expuestos (3.83 \pm 0.11$)$, $\left(0.0002^{*}\right)$, en el área de materiales (puntaje promedio expuestos $5.76 \pm 0.25\left(<0.0001^{\star}\right)$ vs puntaje promedio no expuestos $3.8 \pm 0.24)$; en el área de involucramiento (puntaje promedio expuestos $(3.73 \pm 0.17)$ vs puntaje promedio no expuestos $(2.63 \pm 0.16),\left(<0.0001^{*}\right)$ ); en el área de variedad (puntaje promedio expuestos $(2.98 \pm 0.12)$ vs puntuación promedio no expuestos $\left.(2.02 \pm 0.12),\left(<0.0001^{*}\right)\right)$. Las puntuaciones obtenidas en el HOME en el área de responsividad del cuidador y el área de aceptación no fueron estadísticamente significativas.

Al comparar por nivel de estimulación, hubo diferencias estadísticamente significativas en el nivel de estimulación total. Mientras que en los no expuestos predominó la baja estimulación (49\%), en los expuestos la alta estimulación tuvo un mayor predominio $(35.9 \%)\left(<0.0001^{*}\right)$. También se presentaron diferencias estadísticamente significativas en las áreas de responsividad, aceptación, organización, materiales, involucramiento y variedad, predominando la estimulación media y alta en los expuestos a diferencia de los no expuestos, donde predomina la estimulación baja y media. 


\section{Discusión}

Al evaluar la eficacia de un programa de estimulación temprana sobre pautas de crianza, autoestima, confianza y problemas del desarrollo infantil (Armstrong, Fraser y Morris, 2000), se observó después de cuatro meses de exposición al programa una mejoría en el funcionamiento y en diversos aspectos del ambiente del hogar como la responsividad, organización, uso de materiales e involucramiento de manera positiva con los niños, aspectos en los cuales, de forma global, el sIVIPRODIN demuestra efectos que favorecen el desarrollo del niño y el ambiente en el hogar.

Respecto a los resultados por subescalas, se recomendó que en el área de responsividad, en la cual hay relativamente altos niveles de estimulación baja en ambos grupos, se mejoren las formas de respuesta de los cuidadores a las necesidades del niño y favorezcan su capacidad de dialogar, cantar y platicar con sus hijos, explorar sus emociones e interactuar con ellos, actividades que se ven comprometidas si la condición anímica del cuidador es de ansiedad, depresión o de estrés (Rivera, Figueroa, Soler, Sánchez y Ávila, 2010).

En el área de aceptación se ha fomentado a través del programa que el niño tenga libros adecuados para su edad y que compartan el cuidador y el niño, en un momento del día, la lectura de éstos, ya que en las visitas se observó que era el punto de mayor debilidad en esta subescala. En el área de organización del ambiente se fomentó que los niños exploren nuevos ambientes dentro y fuera del hogar y que tengan un lugar específico para sus juguetes. En la subescala de materiales de juego hay niveles aceptables de estimulación en ambos grupos; se fomenta la construcción en casa de juguetes simples, aunque en muchos casos cuentan con una gran variedad de ellos. En el área de involucramiento se favorece jugar con el niño, hablarle y enseñarle a colaborar e interactuar, y en la subescala de variedad se evalúan las interacciones y se promueve la integración progresiva del niño en las rutinas familiares.

Concluimos que se puede señalar que el SIVIPRODIN tuvo efecto sobre la puntuación global del HOME y una mayor frecuen- 
cia de alta estimulación entre los expuestos, en contraste con los no expuestos que mostraron mayor frecuencia de baja estimulación. La baja estimulación predominó en el grupo de no expuestos en las áreas de responsividad, organización, materiales, involucramiento y variedad, que fue mayor a $25 \%$ en todas, mientras que en el grupo de expuestos esto se revirtió en las subescalas de aceptación, organización, materiales, involucramiento y variedad, donde predominó la alta estimulación.

\section{Referencias}

Ángeles G., G.P. (2011). Evaluación de impacto del programa estancias infantiles para apoyar a madres trabajadoras. Informe final de la evaluación de impacto. Sexto producto, México: Instituto Nacional de Salud Pública (INSP)/Centro de Investigación en Evaluación y Encuesta (CIEE).

Armstrong, K., Fraser, J. y Morris, J. (2000). Promoting Secure Attachment, Maternal Mood and Child Health in a Vulnerable Population: A Randomized Controlled Trial. Pedriatric Child Health, 36, 555-562.

Bronfenbrenner, U. (1999). Environments in Developmental Perspective: Theoretical and Operational Models. En L.F.S, Measuring Environment Across the Life Span: Emerging Methods and Concepts. Washington, Dc: American Psychological Associaton Press.

Rivera, G.R., Figueroa, O.M., Soler, L.K., Sánchez, M. y Ávila, R.H. (2010). Experiencia de la aplicación y criterios para la interpretación de dos versiones del inventario HOME para infantes de 0 a 3 años de vida. Salud Mental, 33(1), 57-66.

Sánchez, C., Rivera, G.R. y Figueroa, O.M. (2009). Promoción del desarrollo infantil temprano: orientaciones y estrategias. México: Editores de Textos Mexicanos.

Tudge, J.R.H. y Mokrova, I. (2009). Uses and Misuses of Bronfenbrenner's Bioecological Theory of Human Development. Journal of Family Theory \& Review, 1, 198-210. 
Tudge, J., Oderob, D., Hoganc, D. y Etz, K. (2003). Relations Between the Everyday Activities of Preschoolers and Their Teachers' Perceptions of Their Competence in the First Years of School. Early Childhood Research Quarterly, 18, 42-64. 